2. Mozaffarian D, Benjamin EJ, Go AS, Arnett DK, Blaha MJ, Cushman M, et al. Executive Summary: Heart Disease and Stroke Statistics-2016 Update: A Report From the American Heart Association. Circulation. 2016;133(4):447-54. Epub 2016/01/27.

3. Chouchani ET, Methner C, Nadtochiy SM, Logan A, Pell VR, Ding SJ, et al. Cardioprotection by S-nitrosation of a cysteine switch on mitochondrial complex I. Nat Med. 2013;19(6):753

\section{TAKOTSUBO SYNDROME ASSOCIATED MIR-16 AND MIR-26A REDUCE CONTRACTILITY OF CARDIOMYOCYTES IN VITRO BY AN INHIBITORY G-PROTEIN DEPENDENT MECHANISM}

${ }^{1}$ Liam Couch* ${ }^{*}{ }^{2}$ Anselm A Derda, ${ }^{2}$ Thomas Thum, ${ }^{1}$ Cesare Terracciano, ${ }^{1}$ Sian Harding. ${ }^{1}$ Imperial College London; ${ }^{2}$ Hannover Medical School

10.1136/heartjnl-2017-311726.197

Introduction Takotsubo syndrome (TTS) is a severe but reversible acute heart failure affecting predominantly post-menopausal women, where ventricular apical akinesis results from extreme adrenaline levels arising with stress. The pleiotropic $\beta^{2} A R$ signals via stimulatory $\left(G_{s}\right)$ and inhibitory $\left(G_{i}\right)$ G-proteins, and whilst $G_{s}$ increases cardiac output, it concomitantly decreases survival. The duality of $\beta^{2} \mathrm{AR}$ is a homeostatic mechanism to limit cardiotoxicity by facilitating a switch to $G_{i}$, serving as cardioprotective despite being cardiodepressive. This is dysregulated in TTS where excess stimulus trafficking to $G_{i}$ results in profound negative inotropy. It is not understood what predisposes patients to TTS, but a microRNA (miR) profile of increased miR-16 and miR-26a has been identified. Given the importance of miRs in other cardiac diseases and that TTS is thought to be causally related to $\hat{\mathrm{I}}^{2} 2 \mathrm{AR}-\mathrm{G}_{\mathrm{i}}$, we hypothesise that these miRs could predispose to the cardiodepression in TTS.

Method miRs were manipulated in adult rat apical cardiomyocytes with blinded transfection using Lipofectamine 3000. Percentage shortening was measured using an Ionoptix system, and pharmacological protocols applied. Calcium transients were obtained using Fluo-4-AM and sarcoplasmic reticulum (SR) calcium content measured with caffeine micro-application. $\mathrm{N}$ numbers displayed as $\mathrm{n} / \mathrm{N}$, where $\mathrm{n} / \mathrm{N}=$ cells/rats.

Results Up-regulation of miR-16 and miR-26a significantly reduced basal contractility $(\mathrm{miR}-16=3.52 \pm 0.34 \%$ versus control $=4.91 \pm 0.46 \% ; \mathrm{n} / \mathrm{N}=30 / 6 ; \quad \mathrm{p}<0.05$ and $\mathrm{miR}-26 \mathrm{a}=2.77$ $\pm 0.21 \%$ versus control $=4.30 \pm 0.43 \% ; \mathrm{n} / \mathrm{N}=50 / 10 ; \mathrm{p}<0.01)$, whereas down-regulation had no effect. miR-16/-26a manipulation did not alter $\hat{\mathrm{I}}^{2} 2 \mathrm{AR}$ response. Inhibiting $\mathrm{Gi}$ with pertussis toxin (PTX) prevented this (miR-16 untreated $=5.08 \pm 0.49 \%$, $\mathrm{n}=22$; versus miR-16 PTX-treated $=8.82 \pm 0.63 \%, \mathrm{n}=20$; $\mathrm{p}<0.001$; and miR-26a untreated $=3.20 \pm 0.29 \%$ versus miR26a PTX-treated $=5.05 \pm 0.48 \% ; \mathrm{n} / \mathrm{N}=30 / 6 ; \mathrm{p}<0.05$ respectively). PTX-treatment did not change contractility of control transfected cells. No synergism was observed with dual miR16/-26a transfection possibly suggesting a unified mechanism. Calcium transient amplitude was decreased with miR-16/-26a up-regulation $(\mathrm{F} / \mathrm{F} 0$ for control $=1.85 \pm 0.04, \mathrm{n} / \mathrm{N}=66 / 4$ versus $\mathrm{miR}-16=1.51 \pm 0.04, \mathrm{n} / \mathrm{N}=37 / 4$ and $\mathrm{miR}-26 \mathrm{a}=1.57 \pm 0.04, \mathrm{n} /$ $\mathrm{N}=26 / 4 ; \mathrm{p}<0.001$ ), along with a concomitant decrease in SR calcium content (caffeine-induced $\mathrm{F} / \mathrm{F} 0$ for control=3.13 $\pm 0.14, \mathrm{n} / \mathrm{N}=32 / 4$ versus $\mathrm{miR}-16=2.13 \pm 0.13, \mathrm{n} / \mathrm{N}=22 / 4$ and $\operatorname{miR}-26 \mathrm{a}=2.57 \pm 0.19, \mathrm{n} / \mathrm{N}=17 / 4 ; \mathrm{p}<0.001$ and $\mathrm{p}<0.05$ respectively).

Conclusion/implication Increased miR-16/-26a reduce basal contractility of cardiomyocytes in vitro, possibly through a shared $\mathrm{G}_{\mathrm{i}}$-dependent mechanism. Decreased calcium transient amplitude is also likely to contribute. This suggests these miRs may be mechanistically involved in TTS, but further work is needed to investigate their specific mechanistic and spatiotemporal involvement.

\section{NITRIC OXIDE PROMOTES INSULIN-INDEPENDENT GLUCOSE UPTAKE AND PRESERVES CARDIAC FUNCTION AND ENERGETICS IN DIABETES}

Klemen Ziberna* ${ }^{1}$ Drew Duglan, ${ }^{2}$ Simona Mafrici, ${ }^{1}$ Jillian Simon, ${ }^{1}$ Craig Lygate, ${ }^{1}$ Keith Channon, ${ }^{1}$ Barbara Casadei, ${ }^{1}$ Ricardo Carnicer. ${ }^{1}$ University of Oxford; ${ }^{2}$ University Magna Graecia of Catanzaro

\subsection{6/heartjnl-2017-311726.198}

Introduction In the presence of diabetes (DM), myocardial glucose uptake and glycolysis are impaired and the heart rapidly adapts to use exclusively fatty acids (FA) for ATP generation. This maladaptation is believed to play a key role in the development of a cardiomyopathy over time. Here, we show that stimulating myocardial nitric oxide synthase (NOS) activity is sufficient to alleviate myocardial metabolic inflexibility, improve energy metabolism and prevent LV dysfunction in DM by increasing myocardial insulin-independent glucose transport.

Methods Myocardial-specific overexpression of GTP cyclohydrolase I (mGCH1) was used to increase both tetrahydrobiopterin (BH4) and NOS activity in cardiomyocytes. Diabetes mellitus (DM) was induced by multiple low-dose streptozotocin injections (vs sham). PCr/ATP ratio was measured in perfused hearts using ${ }^{31} \mathrm{P}-\mathrm{MRS}$, glucose transport estimated by deoxy-glucose uptake, and oxygen consumption rate (OCR) of intact cardiomyocytes using a phosphorescent probe.

Results As expected, sham-injected mGCH1 transgenic hearts had higher BH4 levels and constitutive NOS activity compared with WT. 12 weeks after DM induction, LV dysfunction developed in WT mice but not in mGCH1 mice, in the absence of changes in myocardial $\mathrm{BH} 4$ content and NOS activity in either group. WT diabetic hearts had a lower PCr/ATP ratio $(1.32 \pm 0.1$ vs $1.73 \pm 0.1, \mathrm{p}<0.05, \mathrm{n}=11$ per group) and mitochondrial creatine kinase (CK) activity (1.56 $\pm 0.1 \mathrm{AU}$ vs 1.98 $\pm 0.1 \mathrm{AU}, \mathrm{p}<0.005, \mathrm{n}=10$ per group) when compared with non-diabetic WT mice, consistent with impaired cardiac energetics. By contrast, PCr/ATP and CK activity were preserved in diabetic mGCH1 hearts in the absence of differences in myocardial mitochondrial content.

Myocardial GCH1 overexpression was associated with a higher protein levels of the insulin-independent glucose transporter, GLUT-1 $(\mathrm{p}<0.05, \mathrm{n}=12$ per group), but no changes in GLUT-4 protein. Myocardial glucose transport was $40 \%$ higher in LV myocytes from mGCH1 diabetic mice when compared with WT diabetic mice. This was accompanied by increased myocardial glucose oxidation, as determined by OCR. Pre-incubation of myocytes with inhibitors of NOS-PKG signalling (L-NAME, $1 \mathrm{mmol} / \mathrm{L}$ or Rp8pCPT PET cGMP 10 $\mu \mathrm{mol} / \mathrm{L})$ or GLUT-1 (STF-31, $10 \mu \mathrm{mol} / \mathrm{L}$,) abolished all differences between mGCH and WT diabetic hearts.

Conclusions Our study reveals that a myocardial increase in $\mathrm{BH} 4$ and NOS activity is sufficient to maintain a favourable substrate utilisation and preserve cardiac mitochondrial function in the presence of DM. This work provides new insight into the potential metabolic triggers of diabetic 
cardiomyopathy and suggests exciting new targets for BH4based therapeutics.

\section{HUMAN OXIDISED PHOSPHOLIPID MACROPHAGES HAVE HIGH LIPOPROTEIN HANDLING CAPABILITIES WITHOUT READILY FORMING UNWANTED FOAM CELLS}

${ }^{1}$ Kajus Baidzajevas*, ${ }^{2}$ Eva Hadadi, ${ }^{3}$ Bernett Lee, ${ }^{3}$ Josephine Lum, ${ }^{4}$ lan Sudbery, ${ }^{3}$ Sarah Lai, ${ }^{3}$ Siew Cheng Wong, 'Endre Kiss-Toth, ${ }^{1}$ Heather Wilson. 'Department of Infection, Immunity and Cardiovascular Disease, Medical School, University of Sheffield, S10 2RX, UK; ${ }^{2}$ Laboratoire ESTeam UMRS 935-Batiment A, 7 rue Guy Moquet, F-94800 Villejuif, France; ${ }^{3}$ Singapore Immunology Network (SIgN), A *STAR, Immunos Building, 8A Biomedical Grove, 138648, Singapore; ${ }^{4}$ Department of Molecular Biology and Biotechnology, University of Sheffield, S10 2TN, UK

\subsection{6/heartjnl-2017-311726.199}

Rationale Cardiovascular disease is the leading cause of death world-wide. Macrophages are crucial in regulating the plaque environment, especially the lipid content. However, current characterisation of macrophage phenotypes lipoprotein handling capacity is conflicting and incomplete. We hypothesised that lipoprotein handling differed among distinct macrophage phenotypes due to differential gene and protein expression. We tested this using a range of functional, gene and protein expression assays.

Methodology Monocytes were isolated from healthy donor blood by gradient centrifugation and magnetic selection, then differentiated into macrophages over 7 days using M-CSF. Macrophages were polarised over 24 hour by IFN $\gamma+L P S$, IL4, IL-10, oxPAPC (oxidised phospholipid) and CXCL4, respectively. Unpolarised macrophages were used as controls.

Foam cell formation was determined by Oil-Red-O staining and acLDL uptake was detected by flow cytometry. Cholesterol content and efflux were measured using colorimetric and fluorescent assays. RNA expression was determined by RNAseq and qRT-PCR and cell surface protein expression was measured by flow cytometry.

Results IFNÃ $\square \hat{A}^{3}+$ LPS macrophages did not readily form foam cells (0.23 compared to unpolarised) or process lipoprotein particles, whereas IL-4 and IL-10 polarised macrophages displayed the highest capacity in foam cell formation (1.02 and 1.08 compared to unpolarised) and lipoprotein handling. OxPAPC macrophages exhibited lipoprotein processing capabilities similar to IL-4 and IL-10 macrophages, but did not readily form foam cells (0.22 compared to unpolarised). CXCL4 macrophages displayed intermediate foam cell formation (0.84 compared to unpolarised) and lipoprotein handling capabilities.

Differences in foam cell formation and lipoprotein uptake correlated directly to specific scavenger receptor gene and protein expression. Only IFN $\gamma+$ LPS macrophages had significantly reduced expression of key internal lipoprotein processing genes (q 0.0001). Cholesterol efflux correlated directly to specific $\mathrm{ABC}$ transporter protein, but not RNA expression.

Conclusions. In vitro human macrophage phenotypes differ in foam cell formation and lipoprotein handling capabilities that are associated with differential key gene and protein expression.

\section{THERAPEUTIC RESOLUTION OF PULMONARY ARTERIAL HYPERTENSION (PAH) BY NOVEL SMALL MOLECULE NATURAL PRODUCTS}

$1,2 \mathrm{~N}$ Sharmin* ${ }^{3} \mathrm{H}$ Malik, ${ }^{3} \mathrm{~A}$ Siddiqui, ${ }^{3} \mathrm{H}$ Chowdhury ${ }^{3} \mathrm{~S}$ Kanagenti, ${ }^{4} \mathrm{~A}$ Graham, ${ }^{1} \mathrm{C}$ Wright, ${ }^{5} \mathrm{~N}$ Morrell, ${ }^{3} \mathrm{RC}$ Trembath, ${ }^{1,3,6,7} \mathrm{MT}$ Nasim* ${ }^{*}{ }^{1}$ School of Pharmacy, University of Bradford; ${ }^{2}$ Dept. of Pharmaceutical Technology, University of Dhaka, Bangladesh; ${ }^{3}$ Dept. of Medical and Molecular Genetics, King's College London; ${ }^{4}$ School of Medical Sciences, University of Bradford; ${ }^{5}$ Dept. of Medicine, University of Cambridge; ${ }^{6}$ NIHR Biomedical Research Centre, King's College London, UK; ${ }^{7}$ Centre for Health, Agriculture and Socio-Economic Advancements (CHASA), Lalmonirhat, Bangladesh

\subsection{6/heartjnl-2017-311726.200}

Heterozygous germline mutations in the gene encoding type II bone morphogenetic protein receptor (BMPRII) underlie the majority $(\sim 80 \%)$ of the familial form of pulmonary arterial hypertension (HPAH). ${ }^{1}$ PAH is a devastating cardiovascular disorder caused by narrowing of blood vessels in the lungs. We earlier demonstrated that mutations in BMPR2 impinge upon the BMP signalling pathway and potentiate the TGF- $\beta^{2}$ signalling leading to abnormal proliferation and apoptosis resistance of endothelial and pulmonary arterial smooth muscle cells (PASMCs). ${ }^{2,3}$ No cure for this disorder is known. Traditional therapies aim to improve cardiopulmonary function and were established before recognising the involvement of substantial genetic components of PAH. Hence, there is an urgent need to identify novel compounds capable of providing therapeutic intervention prior to or following the onset of disease.

High-throughput cell based BMP-responsive screens were carried out, which identified several natural compounds as hits. The hit compounds were optimised through a medicinal chemistry programme. These compounds were tested in a number of cell based experiments including reporter assays, gene expression studies and western blot analyses. The compounds CRT-01, CRT-02 and CRT-03 enhanced BMP signalling in BMP responsive reporter assays. Moreover, these compounds were able to induce BMP responsive id1 gene expression and increased the phosphorylation of SMAD1/5 proteins. Furthermore, these compounds inhibited excessive proliferation of PASMCs harbouring a pathogenic BMPR2 mutation. Taken together, this study identified novel compounds eliciting pro-BMP effects which may have experimental and clinical applications in $\mathrm{PAH}$.

\section{REFERENCES}

1. Lane KB, Machado RD, Pauciulo MW, Thomson JR, Phillips JA, Loyd JE, Nichols WC, Trembath RC. Heterozygous germline mutations in BMPR2, encoding a TGF$\hat{i}^{2}$ receptor, cause familial primary pulmonary hypertension. Nature genetics 2000;26(1):81-84

2. Nasim MT. Ghouri A, Patel B, James V, Rudarakanchana N, Morrell NW, Trembath RC. Stoichiometric imbalance in the receptor complex contributes to dysfunctional BMPR-II mediated signalling in pulmonary arterial hypertension. Hum Mol Genet 2008;17(11);1683-94.

3. Nasim MT, Ogo T, Chowdhury HM, Zhao L, Chen CN, Rhodes C, Trembath RC BMPR-II deficiency elicits pro-proliferative and anti-apoptotic responses through the activation of TGFbeta-TAK1-MAPK pathways in PAH. Hum Mol Genet 2012;21(11):2548-58. 